\title{
Recirculation Flow and Pressure Distributions in a Rayleigh Step Bearing
}

\author{
Feng Shen $(\mathbb{D}$, Cheng-Jin Yan, Jian-Feng Dai, and Zhao-Miao Liu \\ College of Mechanical Engineering and Applied Electronics Technology \& Institute for Advanced Mechanics in Engineering, \\ Beijing University of Technology, Beijing 100124, China
}

Correspondence should be addressed to Zhao-Miao Liu; lzm@bjut.edu.cn

Received 26 January 2018; Revised 16 April 2018; Accepted 26 April 2018; Published 21 June 2018

Academic Editor: Enrico Ciulli

Copyright (C) 2018 Feng Shen et al. This is an open access article distributed under the Creative Commons Attribution License, which permits unrestricted use, distribution, and reproduction in any medium, provided the original work is properly cited.

Flow characteristics in the Rayleigh step slider bearing with infinite width have been studied using both analytical and numerical methods. The conservation equations of mass and momentum were solved utilizing a finite volume approach and the whole flow field was simulated. More detailed information about the flow patterns and pressure distributions neglected by the Reynolds lubrication equation has been obtained, such as jumping phenomenon around a Rayleigh step, vortex structure, and shear stress distribution. The pressure distribution of the Rayleigh step bearing with optimum geometry has been numerically simulated and the results obtained agreed with the analytical solution of the classical Reynolds lubrication equation. The simulation results show that the maximum pressure of the flow field is at the step tip not on the lower surface and the increment of the strain rate from Navier-Stokes equation is approximately 49 percent greater than that from Reynolds theory at the step tip. It is also shown that the position of the maximum pressure of the lower surface is a little less than the length of the first region. These results neglected by the Reynolds lubrication equation are important for designing a bearing.

\section{Introduction}

Rayleigh step bearing has been widely used in industry due to its highest load capacity among all other possible bearing geometries. Many researches on improving its load capacity were carried out using an analytical method by solving the classical Reynolds lubrication equation, assuming that the bearing length should be at least 100 times of the film thickness. In 1918, the theory of a step bearing was firstly discussed by Lord Rayleigh [1], determining the optimum geometry with maximum load capacity per unit width for a given film thickness and bearing length. This configuration is now referred to as Rayleigh step bearing.

Because of its high load capacity and cheap manufacturing, the Rayleigh step bearing has been widely used in industry, such as thrust and pad bearings [2-5]. In addition, a series of Rayleigh steps are used in journal bearings to form a grooved bearing with higher performance. Since then, researches into optimum design and fluid dynamic characteristics of this bearing have attracted much attention $[6,7]$. Rahmani et al. [8] comprehensively studied the Rayleigh step slider bearing including the effect of variations of pressure at the boundaries on the optimum parameters. The bearing is also optimized considering the lubricant flow rate, friction force, and friction coefficient. Auloge et al. [9] studied the optimum design of Rayleigh step bearing and determined the relationships between step location and height along with non-Newtonian lubricants. Artiles et al. [10] described the analysis and design of a $50 \mathrm{~mm}$ diameter floating-ring seals with the Rayleigh step lift pads, considering influences of the surface speed, gas pressure, and inertia. Hong et al. [11] investigated the flow of a Newtonian fluid and Bingham fluid in a Rayleigh step bearing.

Through the numerical solutions of the conservation equations of mass and momentum, they found some special jumping phenomena around a Rayleigh step, which are important for designing a bearing and the study of wear characteristics. Zhu [12] investigated both numerically and experimentally the response of a rotor supported on Rayleigh step gas bearing using Galerkin finite element method. Constantinescu et al. [13] analyzed the pressure variation due to fluid inertia effects in Rayleigh step bearings. Faria 
and San Andrés [14] calculated the bearing load capacity, static stiffness coefficients, and frequency-dependent force coefficients for the gas-lubricated plane and Rayleigh step slider bearings using both finite element and finite difference methods. Lee and Kim [15] calculated the air film temperature of Rayleigh step air foil thrust bearing by solving the Reynolds equation and $3 \mathrm{D}$ energy equation with thermohydrodynamic boundary conditions at the top foil, thrust disc, and cooling air plenum. By using the non-Newtonian fluid as a lubricant, the pressure distribution and load capacity for Rayleigh step bearing have also been investigated [11, 16-19].

In previous literature, most researchers used an analytical method by solving the Reynolds lubrication equation, which is derived from fundamental equations governing fluid flows and based on several simplifying assumptions. Thus, the solutions of Reynolds lubrication equation for the pressure distribution, load capacity, and frictional coefficient were obtained through a relatively simple calculation and some detailed flow information on Rayleigh step bearing has been neglected [20]. Only a few investigations used the calculation of the Navier-Stokes equations with finite volume method [11] or infinite element method [12,14]. In recent years, the full Navier-Stokes equations have been increasingly applied to solve some lubrication problems [21-23]. The trend is driven by a growing interest in describing lubricant flow behavior within a whole lubricated system. The Reynolds equation is confined to just the contact region, but the Navier-Stokes equations can be applied throughout the flow field [24, 25]. In order to optimize Rayleigh step bearing design scientifically, it is beneficial to obtain detailed flow information of the whole lubricant flow field [26].

In this study, the flow characteristics in the infinitely wide Rayleigh step bearing have been investigated by solving Navier-Stokes equations and comparing the numerical results with those results obtained by analytical solutions deduced from the Reynolds equation. From the comparison, more detailed flow characteristics and pressure and shear stress distributions have been obtained, and there are some new differences between the results obtained by two different methods. Moreover, the effects of step geometry and velocity of the lower surface on the recirculation flow and pressure and shear stress distributions in the whole flow field are investigated for better control of these bearings.

\section{Analytical Solution}

The geometry of a two-dimensional infinite Rayleigh step slider bearing is shown in Figure 1. There are two opposed surfaces: the upper surface separates the bearing into two regions and the lower surface has a pure tangential sliding motion relative to the upper surface. The one-dimensional Reynolds equation for a steady flow of an incompressible fluid of constant viscosity can be expressed in the following form [27]:

$$
\frac{\partial}{\partial x}\left(h^{3}(x) \frac{\partial p(x)}{\partial x}\right)=6 \mu U_{1} \frac{\partial h(x)}{\partial x}
$$

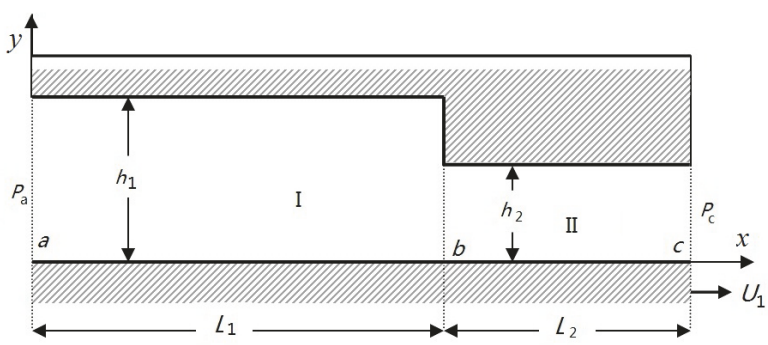

FIGURE 1: The Rayleigh step bearing.

Here $p$ is the oil film pressure, $h$ is the oil film thickness, $\mu$ is the fluid dynamic viscosity, $U_{1}$ is the velocity of the lower surface, and $x$ is the Cartesian position variable.

With reference to Figure 1, the film thickness is given by

$$
\begin{array}{ll}
h(x)=h_{i} & \left(i=1,0 \leq x \leq L_{1}\right) \\
h(x)=h_{i} & \left(i=2, L_{1}<x \leq L\right)
\end{array}
$$

Here $L$ is the characteristic length of the bearing, given by

$$
L=L_{1}+L_{2}
$$

The dimensionless scales of the Rayleigh step bearing can be defined as

$$
\varepsilon=\frac{L_{1}}{L} ; \quad(0<\varepsilon<1)
$$

and

$$
\xi=\frac{h_{1}}{h_{2}} ; \quad(\xi>1)
$$

The boundary conditions for the velocity and the pressure are

$$
\begin{aligned}
& \begin{array}{l}
u=U_{1}, \\
v=0
\end{array} \quad \text { at } y=0 \\
& u=0, \\
& v=0
\end{aligned}
$$

and

$$
\begin{aligned}
& \left.p\right|_{x=c}=p_{0} \\
& \left.p\right|_{x=a}=p_{a}-p_{c}
\end{aligned}
$$

Here $p_{0}$ is the ambient pressure. Double integrating of (1) with respect to $x$, the pressure distribution along the bearing surface can be expressed as

$$
p(x)=6 \mu U_{1} \int_{a}^{c} \frac{1}{h(x)^{2}} d x+c_{1} \int_{a}^{c} \frac{1}{h(x)^{3}} d x+c_{2}
$$


Here $a$ and $c$ are the coordinates of the bearing leading and trailing ends (see Figure 1); $c_{1}$ and $c_{2}$ are integration constants. Substituting boundary conditions (6) and (7) into (8), the integration constants were obtained as

$$
\begin{aligned}
& c_{1}=\frac{\left(p_{a}-p_{c}\right)-6 \mu U_{1} \int_{a}^{c}\left(1 / h(x)^{2}\right) d x}{\int_{a}^{c}\left(1 / h(x)^{3}\right) d x} \\
& c_{2}=0
\end{aligned}
$$

Employing film thickness (2), the pressure distribution for the two regions of the bearing can be expressed in the following forms, respectively:

$$
\begin{aligned}
\left.p(x)\right|_{x \in[a, b]}= & 6 \mu U_{1} \frac{x-a}{h_{1}^{2}}+c_{1} \frac{x-a}{h_{1}^{3}}, \\
\left.p(x)\right|_{x \in[b, c]}= & 6 \mu U_{1}\left[\frac{L_{1}}{h_{1}^{2}}+\frac{x-b}{h_{2}^{2}}\right] \\
& +c_{1}\left[\frac{L_{1}}{h_{1}^{3}}+\frac{x-b}{h_{2}^{3}}\right]
\end{aligned}
$$

In addition, substituting (2) into (9), the integration constant $c_{1}$ was obtained as follows:

$$
c_{1}=\frac{\left(p_{a}-p_{c}\right)-6 \mu U_{1}\left(L_{1} / h_{1}^{2}+L_{2} / h_{2}^{2}\right)}{L_{1} / h_{1}^{3}+L_{2} / h_{2}^{3}}
$$

Using pressure distribution (10), the load capacity of the bearing was calculated as

$$
\begin{aligned}
w= & \int_{a}^{c} p(x) d x \\
= & 3 \mu U_{1}\left[\frac{L_{1}\left(L_{1}+2 L_{2}\right)}{h_{1}^{2}}+\frac{L_{2}^{2}}{h_{2}^{2}}\right] \\
& +\frac{c_{1}}{2}\left[\frac{L_{1}\left(L_{1}+2 L_{2}\right)}{h_{1}^{3}}+\frac{L_{2}^{2}}{h_{2}^{3}}\right]
\end{aligned}
$$

When there is no pressure difference $\left(p_{a}=p_{c}=p_{0}\right)$ between two ends of the bearing, the load capacity of the bearing is entirely supported by the hydrodynamic pressure distribution inside the bearing and the hydrostatic pressure support no longer exists. Equations (10), (11), and (12) will be reduced to a simpler form.

$$
c_{1}^{\prime}=-6 \mu U_{1} h_{1} \frac{L_{1}+L_{2}\left(h_{1}^{2} / h_{2}^{2}\right)}{L_{1}+L_{2}\left(h_{1}^{3} / h_{2}^{3}\right)}
$$

The derivation of (10) is constant:

$$
\begin{aligned}
& \left.\frac{d p(x)}{d x}\right|_{x \in[a, b]}=\frac{6 \mu U_{1}}{h_{1}^{2}}+\frac{c_{1}^{\prime}}{h_{1}^{3}}>0, \\
& \left.\frac{d p(x)}{d x}\right|_{x \in[b, c]}=\frac{6 \mu U_{1}}{h_{2}^{2}}+\frac{c_{1}^{\prime}}{h_{2}^{3}}<0
\end{aligned}
$$

So the maximum pressure $p_{m}$ of the lower surface is at $x=b$; substituting (4), (5), and (13) into (10), $p_{m}$ was obtained as

$$
p_{m}=\frac{6 \mu U_{1} L(a-1) \varepsilon(1-\varepsilon) a^{2}}{h_{1}^{2}\left[\varepsilon+(1-\varepsilon) a^{3}\right]}
$$

where the total load on unit width is

$$
w=\frac{1}{2} L p_{m}=\frac{3 \mu U_{1} L^{2}(a-1) \varepsilon(1-\varepsilon) a^{2}}{h_{1}^{2}\left[\varepsilon+(1-\varepsilon) a^{3}\right]}
$$

\section{Governing Equation and Numerical Methods}

For steady state incompressible flow, the momentum and continuity equations of film region are

$$
\begin{aligned}
u \frac{\partial u}{\partial x}+v \frac{\partial u}{\partial y} & =-\frac{1}{\rho} \frac{\partial p}{\partial x}+\frac{\mu}{\rho}\left(\frac{\partial^{2} u}{\partial x^{2}}+\frac{\partial^{2} u}{\partial y^{2}}\right) \\
u \frac{\partial v}{\partial x}+v \frac{\partial v}{\partial y} & =-\frac{1}{\rho} \frac{\partial p}{\partial y}+\frac{\mu}{\rho}\left(\frac{\partial^{2} v}{\partial x^{2}}+\frac{\partial^{2} v}{\partial y^{2}}\right) \\
\frac{\partial u}{\partial x}+\frac{\partial v}{\partial y} & =0
\end{aligned}
$$

Here $\rho$ is the fluid density, $u$ and $v$ are the velocity, $\mu$ is the fluid viscosity, and $p$ is pressure. The pressure at the inlet and outlet boundaries of the Rayleigh step bearing was set to be atmospheric $\left(10^{5} \mathrm{~Pa}\right)$ and zero velocity gradient in the direction normal to sliding was assumed. This defines a fully developed flow approximation through these boundaries and it is important to set the boundaries relatively far from the region of interest so that they do not influence the numerical solution. The entire domain was fully flooded. At the solid walls, the "no-slip" boundary condition was assumed for the momentum equations. The film region was considered to be a fully developed thin film flow. The bearing working condition is specified by the velocity of the moving wall $\left(U_{1}\right)$, varied from $1 \mathrm{~m} / \mathrm{s}$ to $8 \mathrm{~m} / \mathrm{s}$. The fluid is considered as being incompressible and isothermal with $\mu=0.188 \mathrm{P}_{a} \cdot s$ and $\rho=850 \mathrm{~kg} / \mathrm{m}^{3}$. This approach was adopted because the present work is the first part of a study aimed at enlightening the flow characteristics in the Rayleigh step bearing. Meshes with 210295,334793 , and 5604066 cells were tested. The final refined mesh of the Rayleigh step bearing was built using 334793 cells and the solutions are independent of the refined mesh.

To solve the above equations, a CFD software package CFD-ACE+ (v2010, ESI Corporation) used for multiphysics computational analysis was employed. A finite volume method is utilized to turn the governing partial differential equations (PDE) into a system of algebraic equations and numerically integrated over each of the computational cells using a collocated cell-centered variable arrangement $[28,29]$. A second-order upwind scheme is used for the momentum equations. For pressure calculations, SIMPLEC scheme [30] was used. The equation for pressure correction 


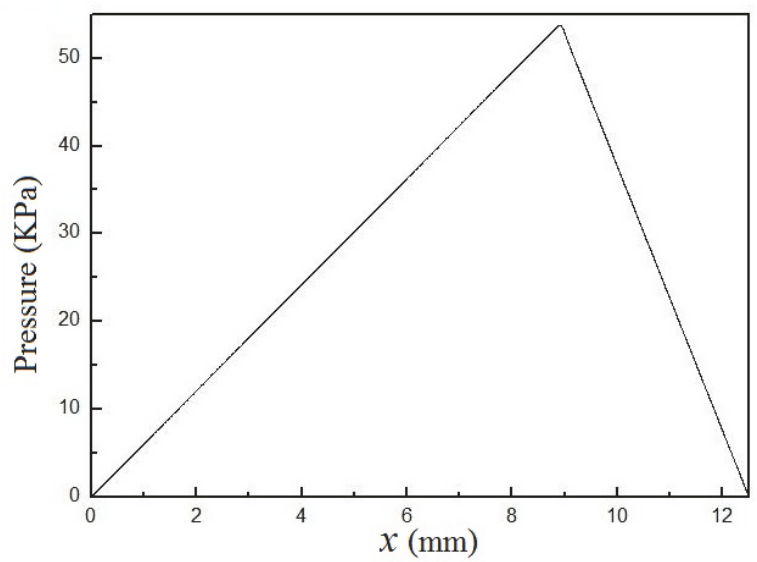

(a)

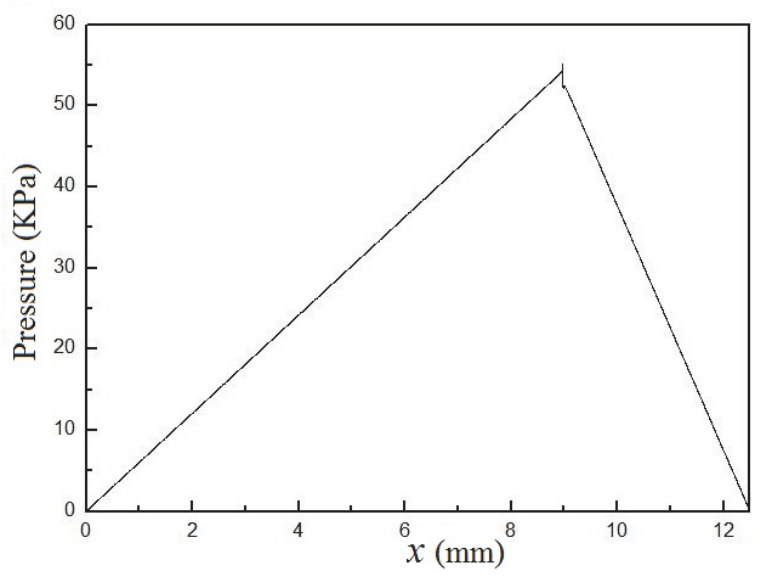

(b)

Figure 2: Pressure distributions on lower (a) and upper surfaces (b).

is obtained from the continuity equation, and the scheme of velocity and pressure calculations is fundamentally iterative in nature. The residuals of continuity and momentum equations are required to be below $10^{-5}$.

\section{Results and Discussion}

The dynamics of flow through a Rayleigh step was investigated. The simulations visualized both the velocity and pressure profiles, while varying system parameters including the depth and length of the step, inlet conditions (pressure and velocity), and shear speed.

4.1. Effects of Length Ratios on Pressure. The geometry and operation conditions are $h_{1}=250 \mu \mathrm{m}, L=12.5 \mathrm{~mm}, h_{2}$ $=133.976 \mu \mathrm{m}, l_{1}=8.975 \mathrm{~mm}, U_{1}=1 \mathrm{~m} / \mathrm{s}$, and $\mu=0.188 P_{a} s$, which are the same as those reported in a previous publication [11]. From the analytic solution of Reynolds lubrication theory, the optimum values of $\varepsilon$ and $\xi$ according to maximum load for a Newtonian fluid are $\varepsilon=0.718$ and $\xi=1.866$ [1], respectively. The maximum pressure $p_{m}$ of $54 \mathrm{KPa}$ can be obtained with the analytic expression of (14). Figure 2 shows the pressure curves on upper and lower surfaces. The maximum pressure

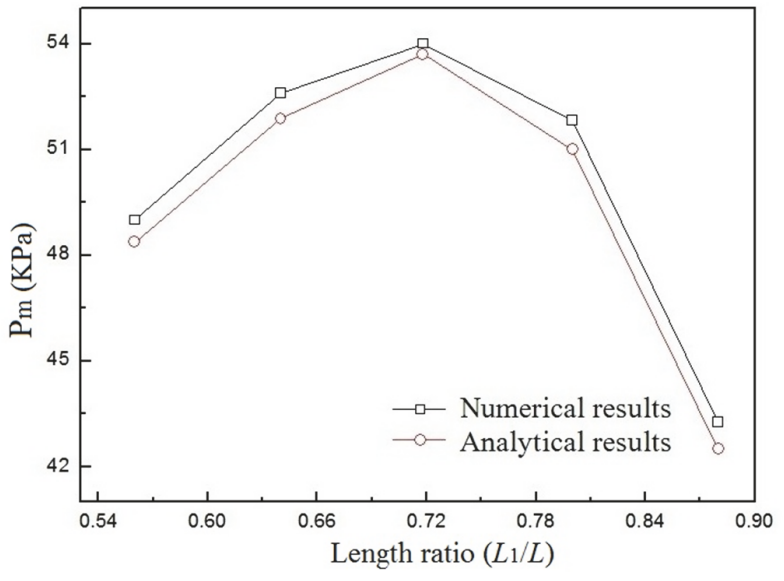

FIgURE 3: $p_{m}$ values with different length ratios $L_{1} / L$.

$\left(p_{m}\right)$ in Figure $2(\mathrm{a})$ is $53.7 \mathrm{KPa}$ close to the analytical value of $54 \mathrm{KPa}$, which is the same as what is reported in a previous publication [11]. Figure 2(b) shows that pressure distribution on the upper surface changes approximately 5.5 percent at the point of $x=b$. The reason is that there is a vortex that appeared near the step and the pressure distribution of flow field is changed significantly, which will be discussed in next section. The results were compared with the simulations of other CFD software (ANSYS-FLUENT, V15.0) in the Supplementary Materials (available here).

The $p_{m}$ values with different length ratios $(\varepsilon=0.56,0.64$, $0.718,0.8$, and 0.88 ) are plotted in Figure 3. The maximum pressure $p_{m}$ can be obtained for $\varepsilon=0.718$ by both analytical and numerical methods. Figure 3 shows that the analytical and numerical results are similar and the validity of the numerical results is further verified.

4.2. Effects of Height Ratios on Pressure. The effects of height ratios on the maximum pressure were simulated. The Rayleigh step parameters are $h_{1}=250 \mu \mathrm{m}, L=12.5 \mathrm{~mm}, \varepsilon=0.718$, $U_{1}=1 \mathrm{~m} / \mathrm{s}$, and $\mu=0.188 P_{a} s$. The $p_{m}$ values with different height ratios $(\xi=1.56,1.786,1.866,2.273,2.778,3.57,5$, and 9.33) are plotted in Figure 4. Figure 4 shows that the maximum pressure $p_{m}$ increases evidently as the value of $\xi$ increases, and both the analytic and numerical results are similar.

Pressure contours around the steps with different height ratios $(\xi=1.866$ and $\xi=9.33$ ) are plotted in Figure 5. Figure 5 shows that the pressure values increase evidently as the value of $\xi$ increases. It is found that the maximum pressure of the flow field is at the step tip instead of being on the lower surface. The maximum pressures of Figures 5(a) and 5(b) are $57 \mathrm{KPa}$ and $154.6 \mathrm{KPa}$, which are higher than the $p_{m}$ values of the lower surface: $53.7 \mathrm{KPa}$ and $152 \mathrm{KPa}$. These details of pressure distribution are not predicted if the Reynolds lubrication equation is used.

4.3. Vortex and Flow Details. The flow characteristics of Rayleigh step bearing with different geometric structures were studied. Some new phenomena like the vortex and 


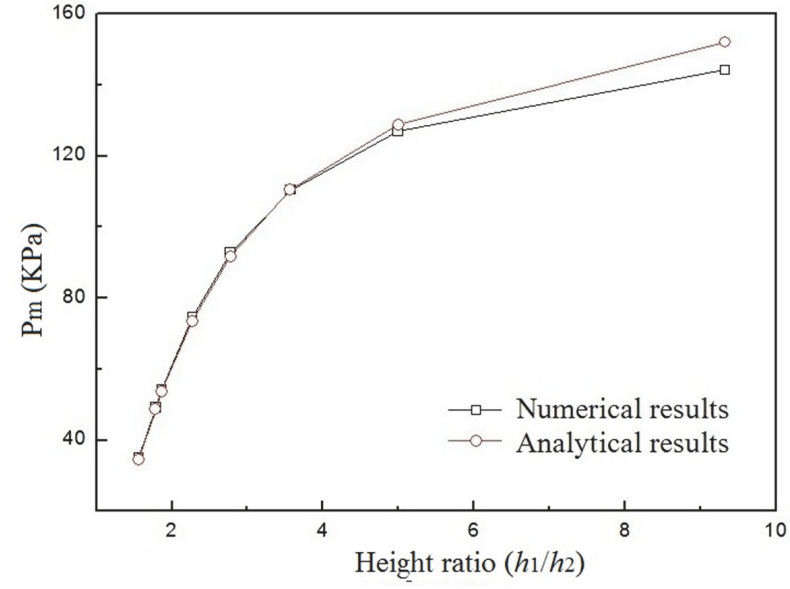

FIGURE 4: $p_{m}$ values with different height ratios $h_{1} / h_{2}$.

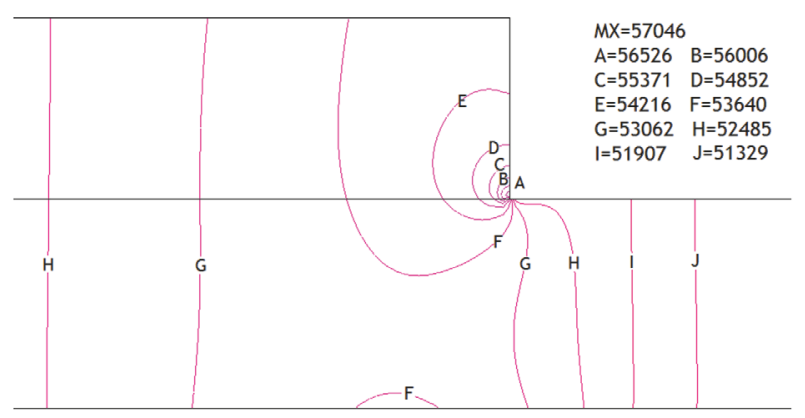

(a)

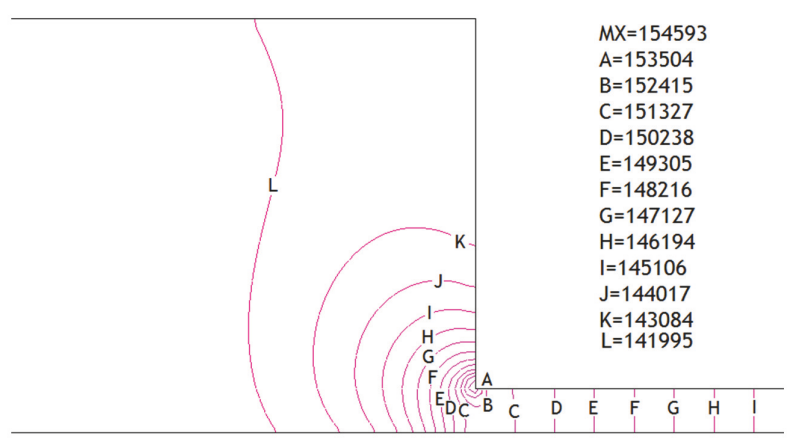

(b)

Figure 5: Pressure contours (Pa) of $\xi=1.866$ (a) and $\xi=9.33$ (b).

reversed flow zone which are not predicted by the Reynolds lubrication equation were investigated. Figure 6 shows the streamlines of different geometric structures in parameters of $U_{1}=1 \mathrm{~m} / \mathrm{s}$ and $\mu=0.188 P_{a}$ s. In Figure 6(a), a reversed flow zone appears near the step with $\varepsilon=0.56$. When $\varepsilon=0.718$ and 0.88 , the reversed flow zone disappeared and vortices are formed, and the vortices become smaller with the increase of length ratios $(\varepsilon)$. In Figure 6(b), two reversed flow zones appear with $\xi=3.57$ and 2.27 , and the center of the reserved flow zone moves rightward with the decrease of the height ratios $(\xi)$. When $\xi=1.7857$, a vortex appears near the step. In

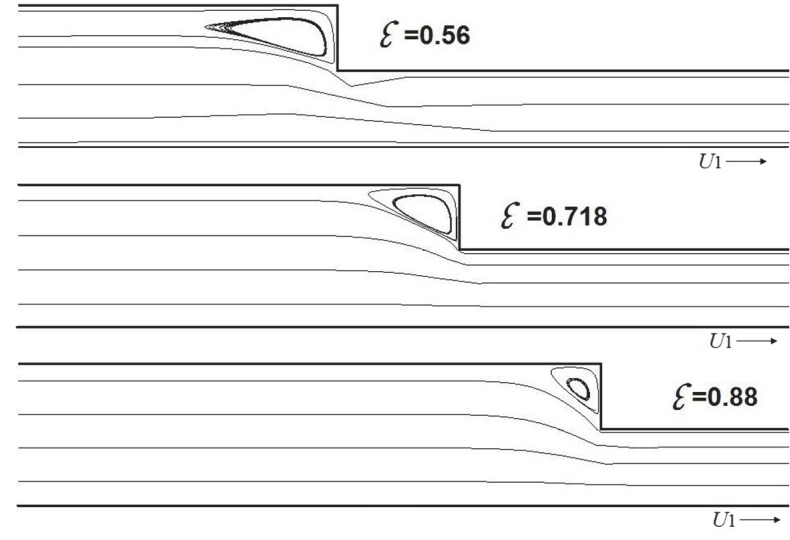

(a)

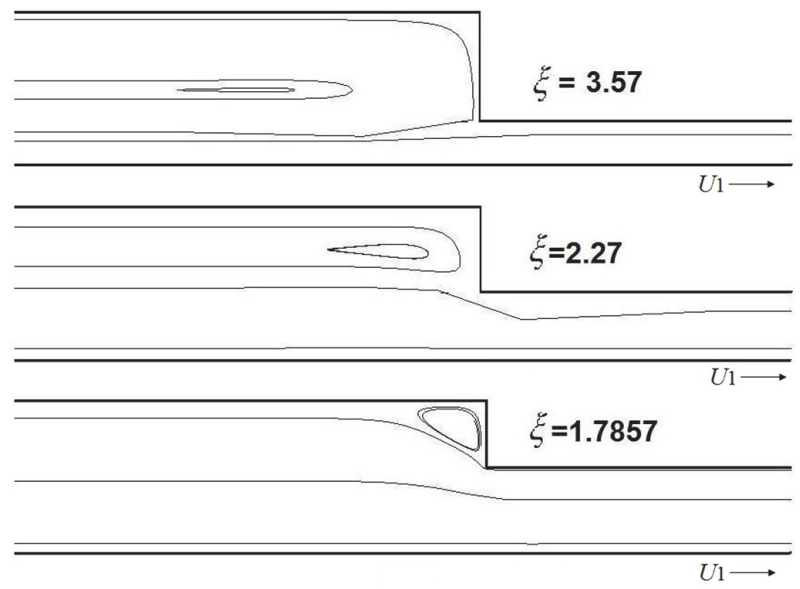

(b)

FIGURE 6: Streamlines of different geometric structures.

the reversed flow zone, the velocity direction near the upper surface is opposite to the velocity of the lower surface $U_{1}$.

The simulation results show that the pressure and shear stress distributions of the Rayleigh step bearing are significantly influenced by the vortex and velocity field. In Figure 7, the strain rates of fluid in Rayleigh step bearing $(\varepsilon=0.718$ and $\xi=1.866)$ are changed obviously near the surfaces. Near the upper surface, the strain rate of fluid in region I is almost zero while that in region II is very high, as shown in Figure 7(a). It is found that the highest value of strain rate appears near the step tip. The strain rate from Navier-Stokes equation is approximately 49 percent greater than that from Reynolds theory at step tip and too strong to be omitted. This jumping phenomenon is not predicted by the Reynolds lubrication equation. Near the lower surface, the strain rate of fluid in region I is higher than that in region II. These results can supply more accurate boundary condition for a better solid analysis of the step tip.

4.4. Effects of $U_{1}$ on Pressure. The velocity of the lower surface $U_{1}$ has important effects on the pressure distribution. Figure 8 shows the lower surface pressures with different $U_{1}$ values in a Rayleigh step bearing with optimum geometry. The pressure 


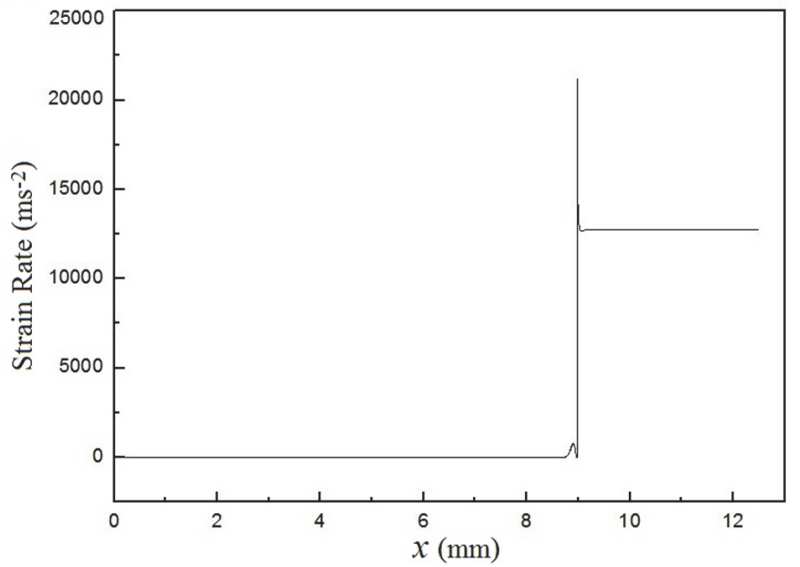

(a)

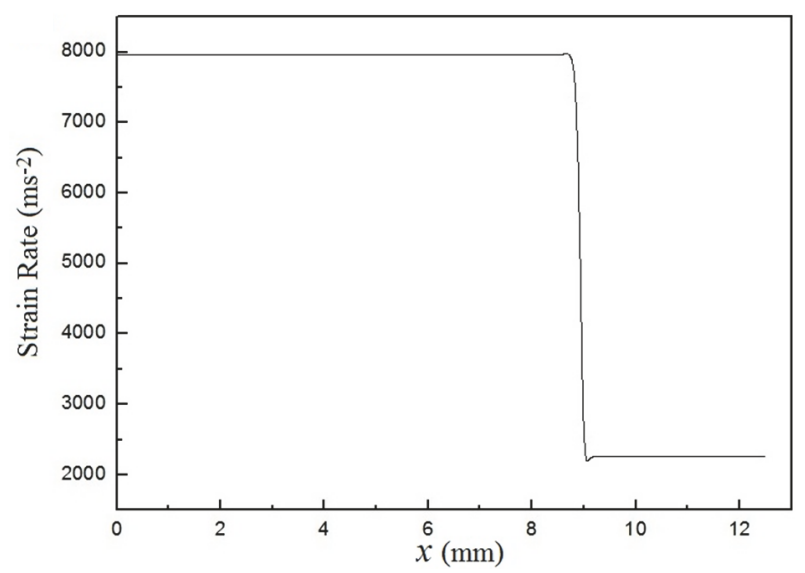

(b)

Figure 7: Strain rates of fluid near the upper (a) and lower (b) surfaces.

values increase evidently as the value of $U_{1}$ increases. The maximum pressure $\left(p_{m}\right)$ of the lower surface was at $x=8.8269$ $\mathrm{mm}$ a little less than $L_{1}=b$, which can be explained in Figure 5. A linear increase of $p_{m}$ values of the lower surface was noted with increasing $U_{1}$ values, shown in Figure 9.

\section{Conclusions}

The current work presents a parametric study of the Rayleigh step bearing with infinite width using two different approaches: (a) analytical solution of the Reynolds equation; (b) the numerical solution of the Navier-Stokes equations by CFD method. The numerical results agree well with the analytical results with parameters of $\varepsilon=0.718$ and $\xi=1.866$. It was shown that the optimum length rate is $\varepsilon=0.718$ with no pressure difference $\left(p_{a}=p_{c}=0\right)$ between two ends of the bearing, and the maximum pressures $\left(p_{m}\right)$ of the lower surface increase evidently as the value of height ratio increases. More detailed information on flow characteristics neglected by Reynolds lubrication equation, such as vortices, reversed flow, shear stress, and pressure distributions, has been obtained. It is found that the maximum pressure of

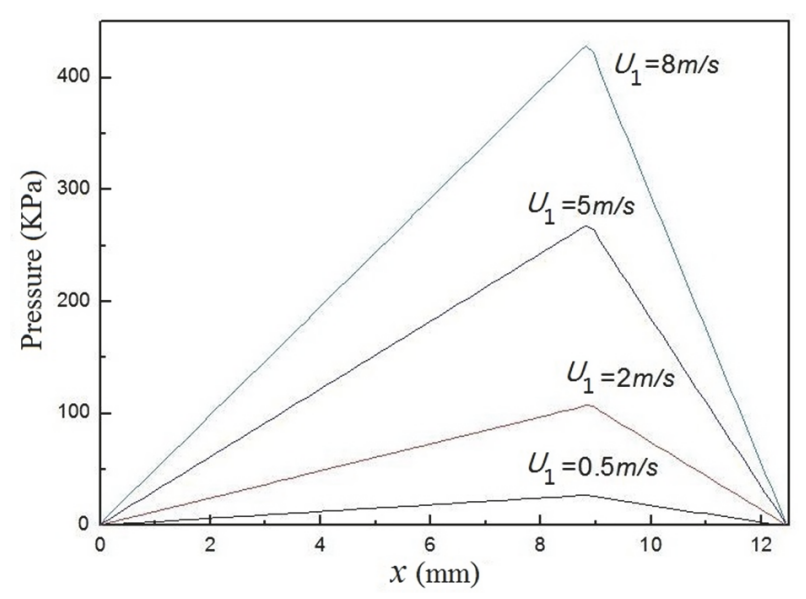

FIGURE 8: Lower surface pressures of different $U_{1}$ values.

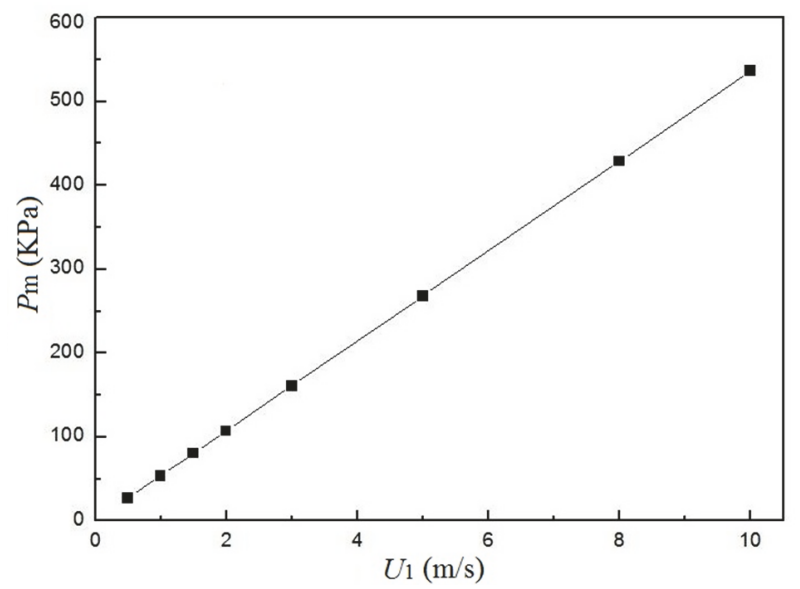

Figure 9: The $p_{m}$ values of the lower surface of different $U_{1}$ values.

the flow field is at the step tip instead of being on the lower surface, and the highest value of strain rate appears near the step tip. The velocity of the lower surface $U_{1}$ has important effects on the pressure distribution. It is also found that the position of the maximum pressure $\left(p_{m}\right)$ of the lower surface is a little less than $x=b$. These results are helpful to supply more accurate flow details in the Rayleigh step bearing when designing an optimum Rayleigh step bearing geometry.

\section{Nomenclature}

a: Coordinate of bearing leading end ( $\mathrm{mm})$

$a_{n b}:$ Link coefficients

$b$ : Coordinate of region I leading end $(\mathrm{mm})$

$c$ : Coordinate of bearing trailing end $(\mathrm{mm})$

$c_{1}$ : Integration constant

$c_{2}$ : Integration constant

$h_{1}$ : Height of region I $(\mu \mathrm{m})$

$h_{2}$ : Height of region II $(\mu \mathrm{m})$

$L: \quad$ Length of the bearing $(\mathrm{mm})$

$L_{1}$ : Length of region I (mm) 
$L_{2}: \quad$ Length of region II $(\mathrm{mm})$

$p$ : $\quad$ Pressure of fluid $(\mathrm{Pa})$

$p_{a}$ : $\quad$ Pressure at bearing leading end $(\mathrm{Pa})$

$p_{c}$ : $\quad$ Pressure at bearing trailing end $(\mathrm{Pa})$

$p_{m}$ : The maximum pressure of the lower surface at $x=b(\mathrm{~Pa})$

$p(x)$ : Pressure distribution along the bearing lower surface $(\mathrm{Pa})$

$U_{1}$ : Velocity of the lower surface $(\mathrm{m} / \mathrm{s})$

$u$ : Velocity in the $x$ direction $(\mathrm{m} / \mathrm{s})$

$v$ : Velocity in the $y$ direction $(\mathrm{m} / \mathrm{s})$

$w$ : Load capacity of the bearing $(\mathrm{N})$

$\mu: \quad$ Viscosity (Pa.s)

$\rho: \quad$ Fluid density $\left(\mathrm{Kg} / \mathrm{m}^{3}\right)$

$\varepsilon: \quad$ Dimensionless length $\left(L_{1} / L\right)$

$\xi$ : Dimensionless height $\left(h_{1} / h_{2}\right)$.

\section{Data Availability}

All data included in this study are available upon request by contact with the corresponding author.

\section{Conflicts of Interest}

The authors declare that they have no conflicts of interest regarding the publication of the paper.

\section{Acknowledgments}

This work was supported by the National Natural Science Foundation of China (11572013), Beijing Municipal Natural Science Foundation (7152012), and Development Project of Beijing Municipal Education Commission (KM201610005002).

\section{Supplementary Materials}

Description of each file of Supplementary Materials: Verification of the results compared with the ANSYS-FLUENT simulations. The results of pressure distributions in Figures 2 and 8 and the results of streamline patterns in Figure 6(a) are simulated and compared with the FLUENT simulations, finding the same results. Figure 1: FLUENT results of pressure distributions on lower surfaces under the same conditions as that in Figure 2(a), finding the deviation of the maximum pressure is $2.4 \%$. Figure 2: FLUENT results of pressure distributions on upper surfaces corresponding to the result of Figure 2(b), finding similar pressure distributions. Figure 3: FLUENT results of lower surface pressures of different U1 values corresponding to the result of Figure 8, finding the same pressure distributions. Figure 4: streamlines in a Rayleigh step bearing with optimum geometry at different U1 values corresponding to Figure 6(a), finding similar flow phenomena. (Supplementary Materials)

\section{References}

[1] L. Rayleigh, "Notes on the theory of lubrication," Philosophical Magazine, vol. 35, pp. 1-12, 1918.
[2] Y. Sato and J. D. Knight, "Performance characteristics of shrouded rayleigh-step and spiral groove viscous pumps," Journal of Tribology, vol. 114, no. 3, pp. 499-503, 1992.

[3] Y. Y. Boldyrev, S. V. Lupulyak, and Y. K. Shinder, "Numerical solution of the variational Rayleigh problem of gas lubrication theory," Journal of Fluid Dynamics, vol. 30, no. 6, pp. 826-832, 1995.

[4] D. G. Farmer and J. J. Shepherd, "Slip flow in the gaslubricated Rayleigh step-slider bearing," Internal Journal of Applied Mechanics and Engineering, vol. 11, pp. 593-608, 2006.

[5] M. B. Dobrica and M. Fillon, "About the validity of Reynolds equation and inertia effects in textured sliders of infinite width," Proceedings of the Institution of Mechanical Engineers, Part J: Journal of Engineering Tribology, vol. 223, no. 1, pp. 69-78, 2009.

[6] M. Oike, M. Nosaka, Y. Watanabe, M. Kikuchi, and K. Kamijo, "Experimental study on high-pressure gas seals for a liquid oxygen turbopump," Tribology Transactions, vol. 31, no. 1, pp. 91-97, 1988.

[7] A. Lipschitz, P. Basu, and R. P. Johnson, "A bi-directional gas thrust bearing," Tribology Transactions, vol. 34, no. 1, pp. 9-16, 1991.

[8] R. Rahmani, A. Shirvani, and H. Shirvani, "Analytical analysis and optimisation of the Rayleigh step slider bearing," Tribology International, vol. 42, no. 5, pp. 666-674, 2009.

[9] J. Y. Auloge, P. Bourgin, and B. Gay, "The optimum design of one-dimensional bearings with non-Newtonian lubricants," Journal of Lubrication Technology, vol. 105, pp. 391-395, 1983.

[10] A. Artiles, W. Shapiro, and H. F. Jones, "Design analysis of Rayleigh-step floating-ring seals," Tribology Transactions, vol. 27, pp. 321-331, 1984.

[11] Y. Hong, D. Chen, J. Wang, and X. Li, "Numerical modeling of a Newtonian and a Bingham fluid in a Rayleigh step bearing," Journal of Tribology, vol. 125, no. 1, pp. 206-210, 2003.

[12] X. Zhu, Experimental response of a rotor supported on Rayleigh step gas bearings [M. S. thesis], Texas A\&M University, College Station, TX, USA, 2004.

[13] V. N. Constantinescu and S. Galetuse, "Pressure Drop Due to Inertia Forces in Step Bearings," Journal of Lubrication Technology, vol. 98, no. 1, p. 167, 1976.

[14] M. T. C. Faria and L. San Andrés, "On the numerical modeling of high-speed hydrodynamic gas bearings," Journal of Tribology, vol. 122, no. 1, pp. 124-130, 2000.

[15] D. Lee and D. Kim, “Three-dimensional thermohydrodynamic analyses of rayleigh step air foil thrust bearing with radially arranged bump foils," Tribology Transactions, vol. 54, no. 3, pp. 432-448, 2011.

[16] K. Yagi and J. Sugimura, "Elastohydrodynamic simulation of Rayleigh step bearings in thin film hydrodynamic lubrication," Tribology International, vol. 64, pp. 204-214, 2013.

[17] A. F. Elkouh and D.-F. Yang, "Flow of a power-law fluid in a rayleigh step," Journal of Tribology, vol. 113, no. 3, pp. 428-433, 1991.

[18] T. H. Leek, S. Lingard, R. J. Atkini, and W. A. Bullough, "An experimental investigation of the flow of an electro-rheological fluid in a rayleigh step bearing," Journal of Physics D: Applied Physics, vol. 26, no. 10, pp. 1592-1600, 1993.

[19] N. B. Naduvinamani, "Non-Newtonian effects of second-order fluids on double-layered porous Rayleigh-step bearings," Japan Society of Fluid Mechanics: Fluid Dynamics Research, vol. 21, no. 6, pp. 495-507, 1997. 
[20] M. Dobrica and M. Fillon, "Reynolds' model suitability in simulating rayleigh step bearing thermohydrodynamic problems," Tribology Transactions, vol. 48, no. 4, pp. 522-530, 2005.

[21] M. J. Braun and M. B. Dzodzo, "Three-Dimensional Flow and Pressure Patterns in a Hydrostatic Journal Bearing Pocket," Journal of Tribology, vol. 119, no. 4, pp. 711-719, 1997.

[22] M. Helene, M. Arghir, and J. Frene, "Numerical three-dimensional pressure patterns in a recess of a turbulent and compressible hybrid journal bearing," Journal of Tribology, vol. 125, no. 2, pp. 301-308, 2003.

[23] J.-S. Kim and K.-W. Kim, "Effects of distance between pads on the inlet pressure build-up on pad bearings," Journal of Tribology, vol. 124, no. 3, pp. 506-514, 2002.

[24] P. Brajdic-Mitidieri, A. D. Gosman, E. Ioannides, and H. A. Spikes, "CFD analysis of a low friction pocketed pad bearing," Journal of Tribology, vol. 127, no. 4, pp. 803-812, 2005.

[25] A. V. Radulescu, "Theoretical Calculus Model for the Finite Length Rayleigh Step Bearings Lubricated with Greases," in Proceedings of the BALTTRIB 2015, Aleksandras Stulginskis University, Akademija, Lithuania, November 2015.

[26] R. Kumar, M. S. Azam, S. K. Ghosh, and H. Khan, "Effect of surface roughness and deformation on Rayleigh step bearing under thin film lubrication," Industrial Lubrication and Tribology, vol. 69, no. 6, pp. 1016-1032, 2017.

[27] Y. Hori, Hydrodynamic Lubrication, Springer-Verlag, Tokyo, Japan, 2006.

[28] D. F. Deng, A numerical and experimental investigation of Taylor flow instabilities in narrow gaps and their relationship to turbulent flow in bearings [Ph.D. thesis], University of Akron, Akron, Ohio, USA, 2007.

[29] F. E. Horvat, Experimental and numerical analysis of flow and pressure fields inside a variable depth single pocket hydrostatic bearing [M. S. thesis], University of Akron, Akron, Ohio, USA, 2008.

[30] J. P. van Doormaal and G. D. Raithby, "Enhancements of the SIMPLE Method for predicting incompressible fluid flows," Numerical Heat Transfer, Part B: Fundamentals, vol. 7, no. 2, pp. 147-163, 1984. 


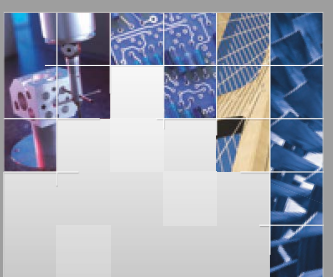

\section{Enfincering}
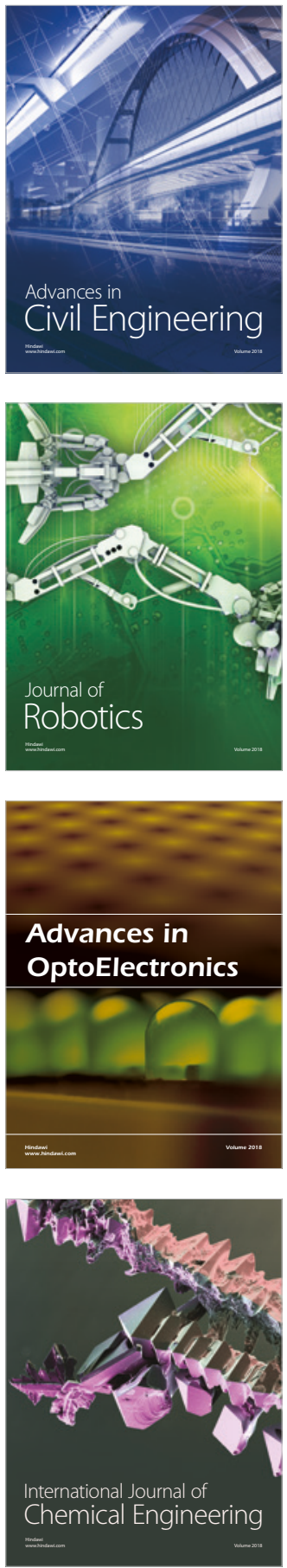

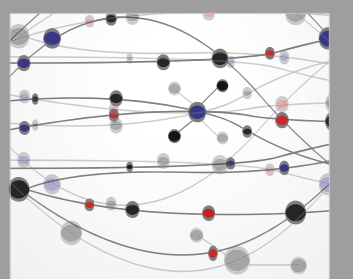

\section{Rotating \\ Machinery}

The Scientific World Journal

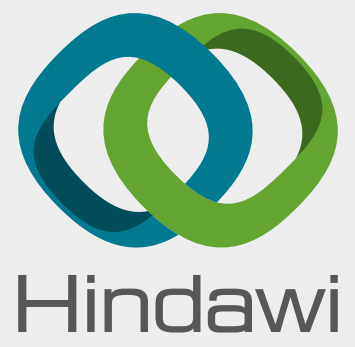

Submit your manuscripts at

www.hindawi.com
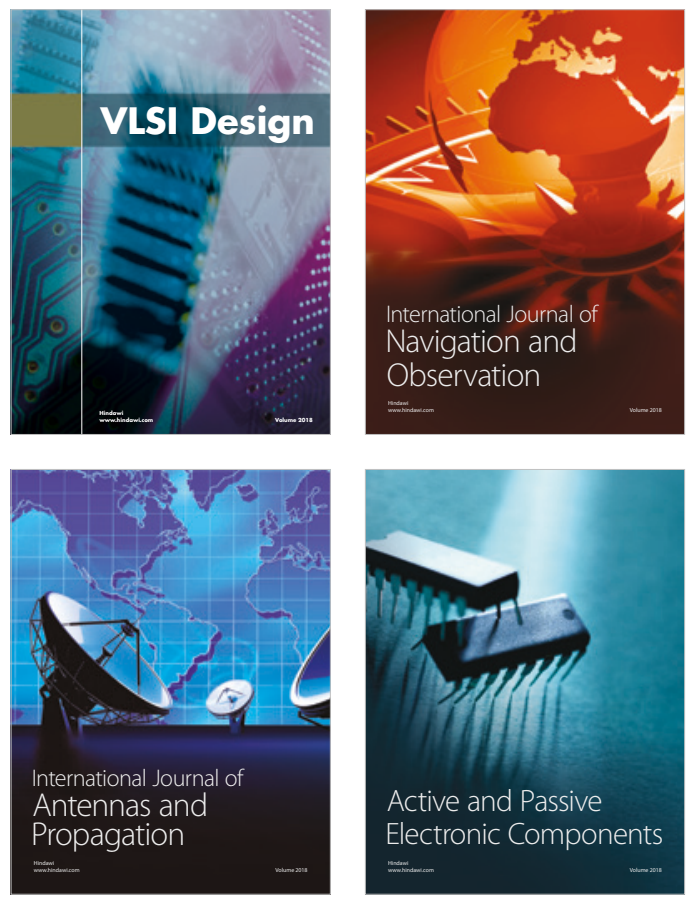
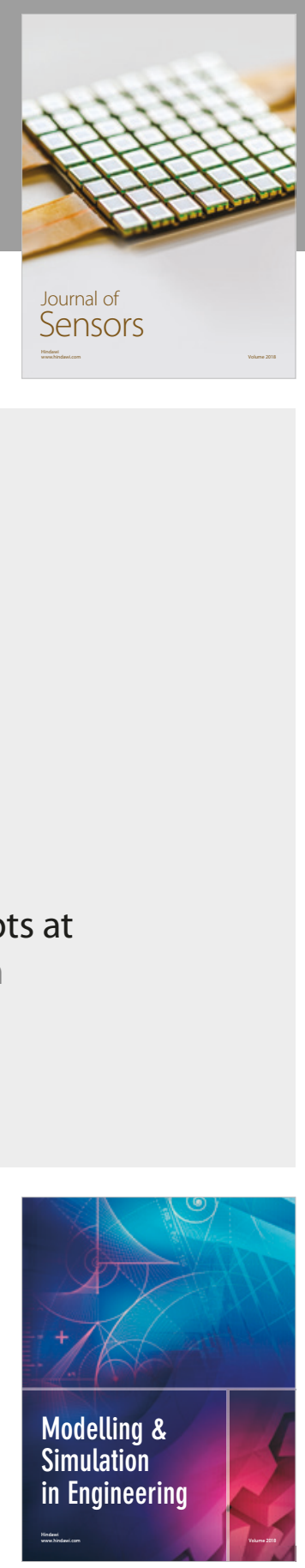

\section{Advances \\ Multimedia}
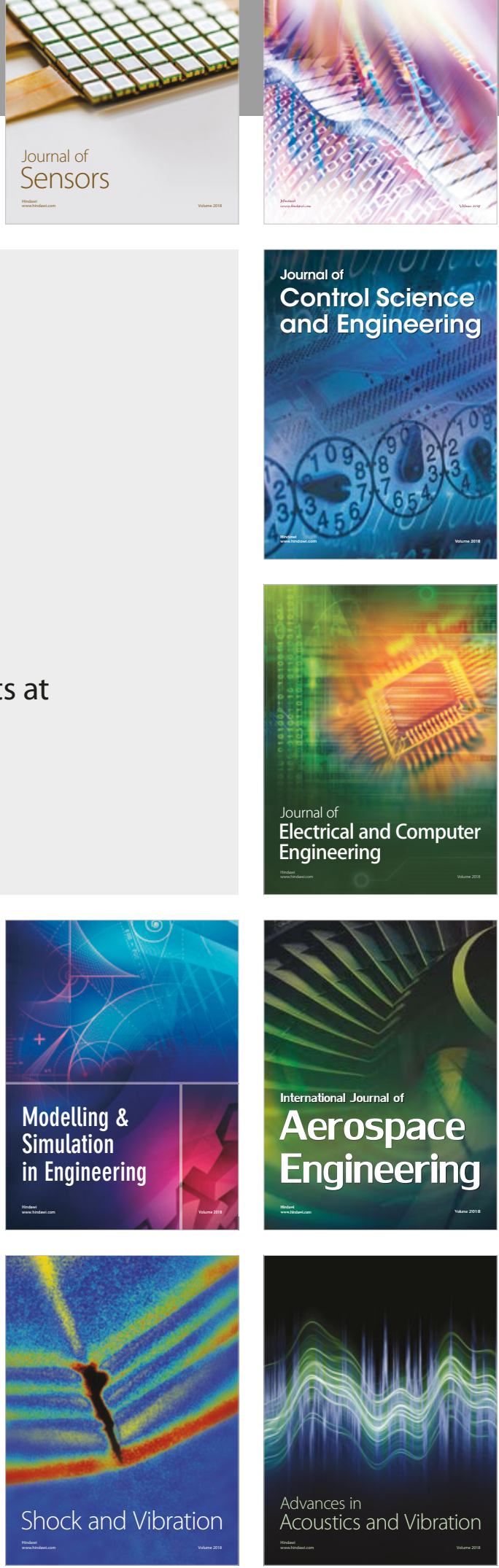